\title{
Large N Chern-Simons with massive fundamental fermions - A model with no bound states
}

\author{
Yitzhak Frishman $^{a}$ and Jacob Sonnenschein ${ }^{b}$ \\ ${ }^{a}$ Department of Particle Physics and Astrophysics, The Weizmann Institute of Science, \\ Rehovot 76100, Israel \\ ${ }^{b}$ The Raymond and Beverly Sackler School of Physics and Astronomy, Tel Aviv University, \\ Ramat Aviv 69978, Israel \\ E-mail: yitzhak.frishman@weizmann.ac.il, cobi@post.tau.ac.il
}

ABSTRACT: In a previous paper [1], we analyzed the theory of massive fermions in the fundamental representation coupled to a $\mathrm{U}(N)$ Chern-Simons gauge theory in three dimensions at level $K$. It was done in the large $N$, large $K$ limits where $\lambda=\frac{N}{K}$ was kept fixed. Among other results, we showed there that there are no high mass "quark anti-quark" bound states. Here we show that there are no bound states at all.

KeYwords: Chern-Simons Theories, Field Theories in Lower Dimensions, Nonperturbative Effects

ArXiv EPRint: 1409.6083 


\section{Contents}

1 Introduction 1

$\begin{array}{llr}2 & \text { The setup } & 2\end{array}$

3 The fermion propagator 3

4 't Hooft like equation for the spectrum of bound states 5

$\begin{array}{llr}5 & \text { No massive bound states } & 8\end{array}$

6 Bound-states in the bosonic theory 9

$\begin{array}{lll}7 & \text { Confronting the results of }[19] & 10\end{array}$

8 Summary and open questions 11

\section{Introduction}

In recent years a major progress has been made in the understanding of large $N$ three dimensional Chern-Simons theory coupled to matter in the fundamental representation [2][15]. Interesting exact results have been derived without the aid of supersymmetry. Among these achievements is the determination of the exact planar free energy of the theory at finite temperature on $\mathcal{R}^{2}$ as a function of the 't Hooft coupling $\lambda=\frac{N}{K}$, where $K$ is the level of the Chern-Simons term. Another property of these theories is the fact that classically in the large $N$ there is an infinite tower of high-spin conserved currents. It was shown in [2] that the divergence of these currents is equal to double and triple trace of currents, that vanish in the large $\mathrm{N}$ limit, as the former is multiplied by $\frac{1}{K}$ and the latter by $\frac{1}{K^{2}}$. In [5] it was shown that in the large $N$ limit the theory of $N$ scalars coupled to $\mathrm{U}(N)$ CS theory at level $K$ is equivalent to the Legendre transform of the theory of $K$ fermions coupled to a $\mathrm{U}(K)$ CS theory at level $N$.

In [2] the fact that one can extract exact results is attributed to the discrete nature of the CS coupling constant, the large $N$ limit, the light-cone gauge and the fact that for the massless case the theory is conformal invariant. The main question addressed in our previous work [1] was to what extent can one decipher the large N CS theory coupled to massive fundamental fermions. Thus our question was essentially whether two of the three ingredients of the CS coupling, large $N$ and the light-cone gauge are enough to enable us to solve it exactly or is conformal symmetry necessary for that. Our answer was that there are interesting physical quantities that can be determined even without conformal invariance. Concretely we had addressed the following three questions: (i) The fermion 
propagator and the thermal free energy. (ii) The hight spin currents and their classical conservations. (iii) The spectrum bound state mesons.

Following [2] we showed that by solving a Schwinger-Dyson equation, the fermion propagator and the partition function at finite temperature can be determined exactly. We generalized the result of [2] to the massive case while using a somewhat different technique. In [3] it was shown that the result of [2] is incomplete and that there is an additional contribution to the thermal free energy from winding modes. The full expression written down in that paper holds for fermions of any mass, with an appropriate modification of the parameters.

We proved that in the large $N$ limit there exists an infinite set of classically conserved high spin currents. The conservation holds classically for high spin currents which are similar to the ones used in the massless case apart from the following replacement

$$
\left(\overleftarrow{D_{\sigma}} \overrightarrow{D^{\sigma}}\right) \rightarrow\left(\overleftarrow{D_{\sigma}} \overrightarrow{D^{\sigma}}\right)-m^{2}
$$

The divergence of these currents is equal to double and triple trace operators, which vanish in the large $N$ limit. This is the same structure as for the conformal invariant setup.

As for the spectrum of bound state mesons, we wrote down, in analogy to the seminal work of 't Hooft on two dimensional QCD, a Bethe-Salpeter equation for the wave function of a "quark anti-quark" bound state. We showed that unlike the two dimensional QCD case, the three dimensional Chern-Simons theory does not admit a confining spectrum. In fact, no high mass bound states exist.

In this paper we extend the latter fact, to show that there are no bound states altogether.

The paper is organized as follows: the next section describes the basic setup of a ChernSimons theory in Euclidean three dimensions in the large $N$ and large level $K$ limits with fixed ratio, coupled to a fermion in the fundamental representation. Section 3 is devoted to the determination of the fermion propagator at zero temperature. In section 4 we write down a 't Hooft-like equation for the bound states of the theory at zero temperature, and transform it to a form closer to the two dimensional case. We then show in section 5 that there are no bound states solutions.

In section 6 we review the connection to the bosonic theory, showing that the correspondence implies no bound states in the fermionic case, as we found. In section 7 we compare with [19], where it was claimed that bound states do exist.

In the last section we summarize our results and present several open questions.

\section{The setup}

The $\mathcal{R}^{3}$ Euclidean action of the $\mathrm{U}(N)$ CS theory coupled to a massive fermion in the fundamental representation is

$$
S=\frac{i K}{4 \pi} \int d^{3} x \operatorname{Tr}\left[A d A+\frac{2}{3} A^{3}\right]+\int d^{3} x \bar{\psi}\left(\gamma^{\mu} D_{\mu}+m_{\text {bare }}\right) \psi
$$

where $A=A^{a} T^{a}, T^{a}$ is a fundamental generator normalized so that $\operatorname{Tr}\left[\left(T^{a}\right)^{2}\right]=\frac{1}{2}$ and $D_{\mu} \psi=\partial_{\mu} \psi-i A_{\mu}^{a} T^{a} \psi$. Note that we set the gauge coupling constant to one. Using 


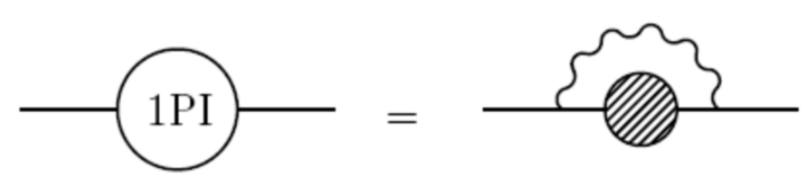

Figure 1. Fermion Self Energy.

light-cone coordinates $x^{+}, x^{-}, x^{3}$ and light-front gauge $A_{-}=0$ the action in momentum space reads

$$
\begin{aligned}
S= & \int \frac{d^{3} p}{(2 \pi)^{3}}\left[-\frac{i K}{2 \pi} \operatorname{Tr}\left[A_{3}(-p) p_{-} A_{+}(p)\right]+\bar{\psi}(-p)\left(i \gamma^{\mu} p_{\mu}+m_{\text {bare }}\right) \psi(p)\right] \\
& -i \int \frac{d^{3} p}{(2 \pi)^{3}} \int \frac{d^{3} q}{(2 \pi)^{3}}\left[\bar{\psi}(-p)\left[\gamma^{+} A_{+}(-q)+\gamma^{3} A_{3}(-q)\right] \psi(p+q)\right]
\end{aligned}
$$

Here

$$
x^{ \pm}=\frac{1}{\sqrt{2}}\left[x^{1} \pm x^{2}\right] \quad A^{ \pm}=\frac{1}{\sqrt{2}}\left[A^{1} \pm A^{2}\right]
$$

It follows from this action that the gauge field propagator takes the form

$$
<A_{\mu}^{a}(p) A_{\nu}^{b}(-q)>=(2 \pi)^{3} \delta(p-q) \delta^{a b} G_{\mu \nu}(p)
$$

where the only non-trivial components of $G_{\mu \nu}(p)$ are

$$
G_{+3}(p)=-G_{3+}(p)=\frac{4 \pi i}{K} \frac{1}{p^{+}}
$$

This translates in configuration space to

$$
<A_{3}(x) A_{+}(0)>=-<A_{+}(x) A_{3}(0)>=\frac{2}{K} \frac{\delta\left(x_{3}\right)}{x^{+}}
$$

\section{The fermion propagator}

The fermion propagator is given by

$$
<\psi^{m}(q) \psi_{n}(-p)>=(2 \pi)^{3} \delta(q-p) \delta_{n}^{m} S(q)=(2 \pi)^{3} \delta(q-p) \delta_{n}^{m} \frac{1}{i \gamma^{\mu} q_{\mu}+m_{\text {bare }}+\Sigma(q)}
$$

where $m, n=1, \ldots N$ and

$$
\Sigma(q)=i \Sigma_{\mu} \gamma^{\mu}+\Sigma_{I} I-m_{\text {bare }} I
$$

The equation for $\Sigma$ takes the form

$$
\Sigma(p)=-i 4 \pi \lambda \int \frac{d^{3} q}{(2 \pi)^{3}} \frac{\gamma^{+} \Sigma_{I}+i I(q+\Sigma(q))_{-}}{\left(q_{\mu}+\Sigma_{\mu}(q)\right)\left(q^{\mu}+\Sigma^{\mu}(q)\right)+\Sigma_{I}(q)^{2}} \frac{1}{(p-q)^{+}}
$$

This is depicted in figure 1. 
Equating the coefficients of the various $\gamma^{\mu}$ matrices it is clear that $\Sigma$ is independent of $p_{3}$ and

$$
\Sigma_{-}=\Sigma_{3}=0 \quad \Sigma_{I}=p_{s} f_{0}\left(\lambda, p_{s}, m_{\text {bare }}\right) \quad \Sigma_{+}=p_{+} g_{0}\left(\lambda, p_{s}, m_{\text {bare }}\right)
$$

with

$$
p_{s}=\sqrt{p_{1}^{2}+p_{2}^{2}}=\sqrt{2}\left|p^{-}\right|=\sqrt{2}\left|p^{+}\right| .
$$

Substituting (3.4) into (3.3) we get the following integral equations for $f_{0}$ and $g_{0}$

$$
\begin{aligned}
g_{0} & =-\frac{4 \pi \lambda}{p^{-}} \int \frac{d^{3} q}{(2 \pi)^{3}} \frac{q_{s} f_{0}}{q_{3}^{2}+q_{s}^{2}\left(1+g_{0}+f_{0}^{2}\right)} \frac{1}{\left(p^{+}-q^{+}\right)} \\
f_{0} p_{s}-m_{\text {bare }} & =4 \pi \lambda \int \frac{d^{3} q}{(2 \pi)^{3}} \frac{q^{+}}{q_{3}^{2}+q_{s}^{2}\left(1+g_{0}+f_{0}^{2}\right)} \frac{1}{\left(p^{+}-q^{+}\right)}
\end{aligned}
$$

To solve for the functions $f_{0}\left(\lambda, p_{s}, m_{\text {bare }}\right)$ and $g_{0}\left(\lambda, p_{s}, m_{\text {bare }}\right)$ we now employ the identity

$$
\frac{\partial}{\partial p^{-}} \frac{1}{p^{+}}=2 \pi \delta^{2}(p)
$$

Applying this to eq. (3.6) we get

$$
\begin{aligned}
\frac{\partial}{\partial p^{-}}\left(p_{s} f_{0}\right) & =\frac{\lambda p^{+}}{p_{s} \sqrt{1+g_{0}+f_{0}^{2}}} \\
\frac{\partial}{\partial p^{-}}\left(p^{-} g_{0}\right) & =-\frac{\lambda f_{0}}{\sqrt{1+g_{0}+f_{0}^{2}}}
\end{aligned}
$$

Multiplying the first with $f_{0} p_{s}$, the second by $p^{+}$and adding, we get zero for the right hand side, thus obtaining

$$
\left(1+\frac{1}{2} p_{s} \frac{\partial}{\partial p_{s}}\right)\left(g_{0}+f_{0}^{2}\right)=0
$$

Which gives the solution

$$
g_{0}+f_{0}^{2}=\frac{m^{2}}{p_{s}^{2}}
$$

The constant of integration comes out to be $m^{2}$, where $m$ is the pole in the full propagator. Using this, the first equation in (3.8) can be integrated, to give

$$
p_{s} f_{0}=\lambda \sqrt{p_{s}^{2}+m^{2}}+C
$$

To determine $\mathrm{C}$, we will evaluate the integral in (3.3) for $p_{s}=0$. Actually, it is enough to evaluate the scalar part. So we have

$$
p_{s} \rightarrow 0:\left(p_{s} f_{0}-m_{\text {bare }}\right) \rightarrow-4 \pi \lambda \int \frac{d^{3} q}{(2 \pi)^{3}} \frac{1}{q^{2}+m^{2}}
$$

The integral is equal to

$$
-\lambda \frac{2}{\pi} \int_{0}^{\infty} d q+\lambda m
$$


Setting the linearly divergent integral to zero, by dimensional regularization, we get that $C=m_{\text {bare }}$, and so

$$
p_{s} f_{0}=\lambda \sqrt{p_{s}^{2}+m^{2}}+m_{\text {bare }}
$$

As for $p_{+} g_{0}$, it follows from the integral equation (3.3) that it vanishes at $p_{s}=0$. This means that $p_{s} f_{0}$ equals $m$ for $p_{s}=0$, entailing

$$
m_{\text {bare }}=m(1-\lambda)
$$

Thus the functions $f_{0}$ and $g_{0}$ and hence the non-trivial components of $\Sigma$ are given by

$$
\begin{aligned}
& p_{s} f_{0}\left(\lambda, p_{s}, m_{\text {bare }}\right)=m+\lambda\left[\sqrt{p_{s}^{2}+m^{2}}-m\right] \\
& p_{s}^{2} g_{0}\left(\lambda, p_{s}, m_{\text {bare }}\right)=-\lambda\left[2 m(1-\lambda)\left[\sqrt{p_{s}^{2}+m^{2}}-m\right]+\lambda p_{s}^{2}\right]
\end{aligned}
$$

Note that we got this solution without solving for the integrals, just by their form and their values at $p=0$.

It follows from (3.16) that $\Sigma$ takes the form

$$
\Sigma(p)=i p_{+}\left[-\lambda^{2}-2 \lambda\left(\frac{m_{\text {bare }}}{p_{s}}\right)\left(\sqrt{1+\frac{m^{2}}{p_{s}^{2}}}-\frac{m}{p_{s}}\right)\right] \gamma^{+}+\lambda p_{s} \sqrt{1+\frac{m^{2}}{p_{s}^{2}}} I
$$

Thus the coefficient of the unit matrix in $\Sigma$, which was for the massless case $\lambda p_{s}$, is still linear in $\lambda$ but there is a re-scaling of $p_{s} \rightarrow p_{s} \sqrt{1+\frac{m^{2}}{p_{s}^{2}}}$. The coefficient of $\gamma^{+}, i p_{+} g_{0}$, which for the massless case was $-i p_{+} \lambda^{2}$, is determined in the massive case from the relation (3.10).

It is easy to check that for the massless limit these results go back to

$$
f_{0}=\lambda \quad g_{0}=-\lambda^{2}
$$

To summarize the propagator of the massive fermion takes the form

$$
S(q)=-\frac{i q_{-} \gamma^{-}+i q_{+}\left(1-g_{0}\right) \gamma^{+}+i q_{3} \gamma^{3}-f_{0} q_{s}}{q^{2}+m^{2}}
$$

\section{4 't Hooft like equation for the spectrum of bound states}

In the conformal setup when the fermions are massless a natural question to address is the spectrum of dimensions of the primary operators and their descendants. The primaries are the operator $\bar{\psi} \psi$ and the tower of symmetric traceless currents $J_{\mu_{1}, \ldots \mu_{s}}^{(s)}$, which are constructed from a fermion anti-fermion bilinear sandwiching derivatives and a gamma matrix. The analysis of the spectrum of dimensions was carried out in [2]. The analogous question for the massive theory is the mass spectrum of bound states. The latter can be built in the same way as in the conformal theory. Here we will discuss a special class of the mesonic bound states. Note also that since the theory is invariant under local $\mathrm{U}(N)$ symmetry, and not only $\mathrm{SU}(N)$, baryon bound states are not gauge invariant. ${ }^{1}$ We address the question of the spectrum of masses only at zero temperature.

\footnotetext{
${ }^{1}$ Note, however, that at large $N$ the $\mathrm{U}(1)$ part is down by $\frac{1}{N}$.
} 

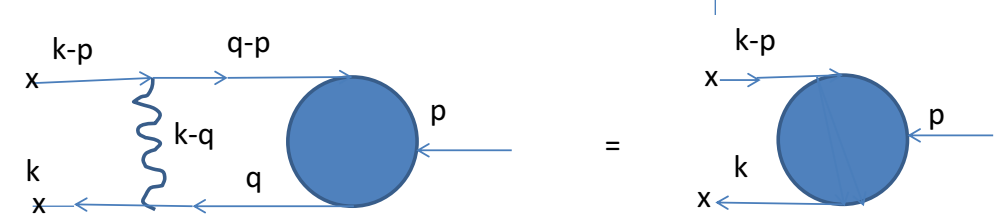

Figure 2. Schwinger-Dyson equation for the fermion anti-fermion bound state.

The spectrum of fermion anti-fermion bound states of two dimensional QCD in the planar limit was solved in the seminal work of 't Hooft ([16]; an elaborated review can be found in the book [17]). Since like in that work, here we are also using (i) light-front coordinates, (ii) light-cone gauge and (iii) the planar limit, it calls for the use of a similar approach to the one used in [16] for our system. The key player is the bound state "wavefunction" or the "blob" which is the Fourier transform of the matrix element of the operator $\psi(x) \bar{\psi}(0)$ between the vacuum and the meson states,

$$
\phi(k, p)=\int \frac{d^{3} x}{(2 \pi)^{3}} e^{i k x}<\operatorname{meson}(p)|T \psi(x) \bar{\psi}(0)| 0>
$$

To determine the "wave-function" one has to solve a Bethe-Salpeter which is depicted in figure 2.

For gauge invariance we need also a factor of $e^{i \int_{0}^{x} A_{\mu}(y) d y^{\mu}}$ between $\psi$ and $\bar{\psi}$. In the gauge $A_{-}=0$, only the integrals over $A_{+} d y^{+}$and $A_{3} d y^{3}$ will appear. As we will integrate over $k_{3}$ (see following (4.11)), this means $x^{3}=0$, leaving us with only an integral over $A_{+} d y^{+}$. The discussion after eq. (4.13) indicates that actually $x^{+}=0$. Thus we discuss here directly the matrix element (4.1).

Note that the correlator in the definition of $\phi(p, k)$ includes the operators $\psi$ and $\bar{\psi}$ at different points $\psi(x) \bar{\psi}(0)$. Expanding $\psi(x)$ around $x=0$ we get bilinear operators of $\psi$ and $\bar{\psi}$ with any arbitrary number of derivatives $\left.\left(\partial_{\mu_{1}} \ldots \partial_{\mu_{n}} \psi\right) \bar{\psi}\right|_{x=0}$. Thus the blob describes a bound state of a quark and an anti-quark with all possible orbital momenta. As for the internal spin, $\phi(p, k)$ is a $2 \times 2$ matrix, so it includes the spin zero and one components, and those are all the Dirac bilinear combinations in 3 dimensions.

To determine the masses of the bound states, we will have to go back to Minkowski space. But let us first continue in Euclidean space.

The integral equation reads

$$
\phi(k, p)=-\frac{\lambda}{2 \pi^{2}} S(k) \int \frac{d^{3} q}{\left(k^{+}-q^{+}\right)}\left[\gamma^{+} \phi(q, p) \gamma^{3}-\gamma^{3} \phi(q, p) \gamma^{+}\right] S(k-p)
$$

Using (3.7) we can transform the integral equation into the following differential equation

$$
\frac{\partial}{\partial k^{-}}\left[S^{-1}(k) \phi(k, p) S^{-1}(k-p)\right]=\frac{\lambda}{\pi}\left[\gamma^{3} \tilde{\phi}(\tilde{k}, p) \gamma^{+}-\gamma^{+} \tilde{\phi}(\tilde{k}, p) \gamma^{3}\right]
$$

where $\tilde{k}$ is the vector $\left(k_{1}, k_{2}\right)$, and

$$
\tilde{\phi}(\tilde{k}, p)=\int d k^{3} \phi(p, k)
$$


Next we expand the blob in terms of the coefficients of $\gamma^{\mu}$ and $I$ similar to (3.2)

$$
\phi=\phi_{-} \gamma^{-}+\phi_{+} \gamma^{+}+\phi_{3} \gamma^{3}+\phi_{s} I
$$

which gives

$$
\gamma^{3} \phi \gamma^{+}-\gamma^{+} \phi \gamma^{3}=2\left[\phi_{s} \gamma^{+}-\phi_{-} I\right]
$$

and similarly for $\tilde{\phi}$.

Thus the right hand side of (4.2) involves $\phi_{s}$ and $\phi_{-}$only. This results in two coupled integral equations for $\phi_{s}$ and $\phi_{-}$, with $\phi_{+}$and $\phi_{3}$ determined from $\phi_{s}$ and $\phi_{-}$.

The integral equation now implies

$$
\begin{aligned}
\frac{\pi^{2}}{\lambda} \phi_{s}(k, p)= & {\left[a_{-}\left(b_{s}-b_{3}\right)+b_{-}\left(a_{3}+a_{s}\right)\right] \int \frac{d^{3} q}{\left(k^{+}-q^{+}\right)} \phi_{s}(q, p) } \\
& -\left[a_{-} b_{+}+a_{+} b_{-}+a_{3} b_{3}+a_{s} b_{s}\right] \int \frac{d^{3} q}{\left(k^{+}-q^{+}\right)} \phi_{-}(q, p) \\
\frac{\pi^{2}}{\lambda} \phi_{-}(k, p)= & {\left[2 a_{-} b_{-}\right] \int \frac{d^{3} q}{\left(k^{+}-q^{+}\right)} \phi_{s}(q, p) } \\
& -\left[a_{-}\left(b_{3}+b_{s}\right)+b_{-}\left(a_{s}-a_{3}\right)\right] \int \frac{d^{3} q}{\left(k^{+}-q^{+}\right)} \phi_{-}(q, p)
\end{aligned}
$$

where

$$
a_{s}=-\frac{f_{0} k_{s}}{k^{2}+m^{2}} \quad a_{-}=\frac{i k_{-}}{k^{2}+m^{2}} \quad a_{+}=\frac{i k_{+}\left(1-g_{0}\right)}{k^{2}+m^{2}} \quad a_{3}=\frac{i k_{3}}{k^{2}+m^{2}}
$$

and $\left(b_{s}, b_{-}, b_{+}, b_{3}\right)$ are given similarly with the same expressions but with $k-p$ replacing $k$.

Let us choose now the frame

$$
p=\left(0,0, p_{3}\right)
$$

Then

$$
\begin{aligned}
a_{-} & =\frac{i k_{-}}{\left[k^{2}+m^{2}\right]} & b_{-} & =\frac{i k_{-}}{\left[k_{s}^{2}+\left(k_{3}-p_{3}\right)^{2}+m^{2}\right]} \\
a_{+} & =\frac{i k_{+}\left[1-g_{0}\left(k_{s}\right)\right]}{\left[k^{2}+m^{2}\right]} & b_{+} & =\frac{i k_{+}\left[1-g_{0}\left(k_{s}\right)\right]}{\left[k_{s}^{2}+\left(k_{3}-p_{3}\right)^{2}+m^{2}\right]} \\
a_{s} & =-\frac{k_{s} f_{0}\left(k_{s}\right)}{k^{2}+m^{2}} & b_{s} & =-\frac{k_{s} f_{0}\left(k_{s}\right)}{k_{s}^{2}+\left(k_{3}-p_{3}\right)^{2}+m^{2}} \\
a_{3} & =\frac{i k_{3}}{k^{2}+m^{2}} & b_{3} & =\frac{i\left(k_{3}-p_{3}\right)}{k_{s}^{2}+\left(k_{3}-p_{3}\right)^{2}+m^{2}}
\end{aligned}
$$

The integral equations become

$$
\begin{aligned}
\frac{\pi^{2}}{\lambda} \phi_{s}(k, p)= & -\frac{k^{+}\left[p_{3}+2 i k_{s} f_{0}\left(k_{s}\right)\right]}{\left[k^{2}+m^{2}\right]\left[k_{s}^{2}+\left(k_{3}-p_{3}\right)^{2}+m^{2}\right]} \int \frac{d^{3} q}{\left(k^{+}-q^{+}\right)} \phi_{s}(q, p) \\
& +\frac{k_{s}^{2}+k_{3}\left(k_{3}-p_{3}\right)-m^{2}}{\left[k^{2}+m^{2}\right]\left[k_{s}^{2}+\left(k_{3}-p_{3}\right)^{2}+m^{2}\right]} \int \frac{d^{3} q}{\left(k^{+}-q^{+}\right)} \phi_{-}(q, p) \\
\frac{\pi^{2}}{\lambda} \phi_{-}(k, p)= & -\frac{\left(k^{+}\right)^{2}}{\left[k^{2}+m^{2}\right]\left[k_{s}^{2}+\left(k_{3}-p_{3}\right)^{2}+m^{2}\right]} \int \frac{d^{3} q}{\left(k^{+}-q^{+}\right)} \phi_{s}(q, p) \\
& +\frac{k^{+}\left[2 i k_{s} f_{0}\left(k_{s}\right)-p_{3}\right]}{\left[k^{2}+m^{2}\right]\left[k_{s}^{2}+\left(k_{3}-p_{3}\right)^{2}+m^{2}\right]} \int \frac{d^{3} q}{\left(k^{+}-q^{+}\right)} \phi_{-}(q, p)
\end{aligned}
$$


We can now perform an integration over $k_{3}$ on both sides. Note that the integrals on the right-hand-sides do not depend on $k_{3}$. Thus the integration can be done directly, yielding an equation for $\tilde{\phi}(\tilde{k}, p)=\int d k_{3} \phi(k, p)$ with $\tilde{k}=\left(k_{+}, k_{-}\right)$.

To find the bound states, we have to go to Minkowski space, by analytic continuation to $p_{3}=i M_{b}$. The solutions of the integral equation should provide us with the masses of the bound states $M_{b}$.

To perform the integrals over $k_{3}$, we will make use of the following integrals

$$
\begin{aligned}
\int \frac{d k_{3}}{\left[k_{s}^{2}+k_{3}^{2}+m^{2}\right]\left[k_{s}^{2}+\left(k_{3}-p_{3}\right)^{2}+m^{2}\right]} & =\frac{2 \pi}{\sqrt{k_{s}^{2}+m^{2}}} \frac{1}{\left[p_{3}^{2}+4\left(k_{s}^{2}+m^{2}\right)\right]} \\
\int d k_{3} \frac{k_{3}\left(k_{3}-p_{3}\right)}{\left[k_{s}^{2}+k_{3}^{2}+m^{2}\right]\left[k_{s}^{2}+\left(k_{3}-p_{3}\right)^{2}+m^{2}\right]} & =\frac{2 \pi \sqrt{k_{s}^{2}+m^{2}}}{\left[p_{3}^{2}+4\left(k_{s}^{2}+m^{2}\right)\right]}
\end{aligned}
$$

The integral equations, after the $k_{3}$ integration, become

$$
\begin{aligned}
\frac{\pi}{2 \lambda k^{+}} \sqrt{k_{s}^{2}+m^{2}}\left[p_{3}^{2}+4\left(k_{s}^{2}+m^{2}\right)\right] \tilde{\phi}_{s}\left(\tilde{k}, p_{3}\right)= & -\left[2 i k_{s} f_{0}\left(k_{s}\right)+p_{3}\right] \int \frac{d^{2} \tilde{q}}{\left(k^{+}-q^{+}\right)} \tilde{\phi}_{s}\left(\tilde{q}, p_{3}\right) \\
& +4 k^{-} \int \frac{d^{2} \tilde{q}}{\left(k^{+}-q^{+}\right)} \tilde{\phi}_{-}\left(\tilde{q}, p_{3}\right) \\
\frac{\pi}{2 \lambda k^{+}} \sqrt{k_{s}^{2}+m^{2}}\left[p_{3}^{2}+4\left(k_{s}^{2}+m^{2}\right)\right] \tilde{\phi}_{-}\left(\tilde{k}, p_{3}\right)= & -k^{+} \int \frac{d^{2} \tilde{q}}{\left(k^{+}-q^{+}\right)} \tilde{\phi}_{s}\left(\tilde{q}, p_{3}\right) \\
& +\left[2 i k_{s} f_{0}\left(k_{s}\right)-p_{3}\right] \int \frac{d^{2} \tilde{q}}{\left(k^{+}-q^{+}\right)} \tilde{\phi}_{-}\left(\tilde{q}, p_{3}\right)
\end{aligned}
$$

Note that, in the integrals on the right-hand-sides, what appears is $\int \frac{d q^{+}}{k^{+}-q^{+}} \int d q^{-} \tilde{\phi}(\tilde{q}, p)$. The integration over $d q^{-}$indicates that it is actually $x_{-}=0$, and hence no need to have an integral of the form $i \int_{0}^{x_{-}} A_{+}(y) d y_{-}$in the Wilson line between $\psi$ and $\bar{\psi}$.

It is amusing to note, that although it is $x_{-}=0$ on the right-hand-sides, as follows from the integration $d q^{-}$, there is no such action on the left-hand-side. However, if there is a bound state, it definitely has to appear in these equations, as it must couple to quark anti-quark combination.

\section{$5 \quad$ No massive bound states}

Let us examine the equations (4.13), looking for possible solutions, namely bound states.

To this end, let us first examine the behavior at large $k$ components. This gives,

$$
\begin{aligned}
\frac{\pi}{\lambda} k_{s}^{2} \tilde{\phi}_{s}\left(\tilde{k}, p_{3}\right) & \sim-i \lambda \int d^{2} \tilde{q} \tilde{\phi}_{s}\left(\tilde{q}, p_{3}\right)+\frac{2 k^{-}}{\sqrt{k}^{2}} \int d^{2} \tilde{q} \tilde{\phi}_{-}\left(\tilde{q}, p_{3}\right) \\
\frac{\pi}{\lambda} k_{s}^{2} \tilde{\phi}_{-}\left(\tilde{k}, p_{3}\right) & \sim-\frac{k^{+}}{2 \sqrt{k}^{2}} \int d^{2} \tilde{q} \tilde{\phi}_{s}\left(\tilde{q}, p_{3}\right)+i \lambda \int d^{2} \tilde{q} \tilde{\phi}_{-}\left(\tilde{q}, p_{3}\right) \\
k_{1}, k_{2} & \rightarrow \infty
\end{aligned}
$$

We have used also equation (3.16). One may wonder whether the region of $q^{+}$near $k^{+}$in the integrals of the right-had sides in (4.13) can change the asymptotic behavior of 
$k^{+}$due to a possible singularity. In fact there is no such a singularity. The proof of the absence of singularity is as follows. Consider the integral

$$
G_{+}(\tilde{k}, \ldots)=\int \frac{d^{2} \tilde{q}}{\left(k^{+}-q^{+}\right)} F(\tilde{q}, \ldots)
$$

where $F(\tilde{q}, \ldots)$ is a non-singular scalar function and the ... represent other variables.

We would like to regularize the denominator, by

$$
\frac{1}{\left(k^{+}-q^{+}\right)} \rightarrow \frac{\left(k^{-}-q^{-}\right)}{\left[(\tilde{k}-\tilde{q})^{2}+\epsilon^{2}\right]}
$$

We now get, for the function $G_{+}$, that

$$
G_{+}(\tilde{k}, \ldots)=\int d^{2} \tilde{q} \frac{\left(k^{-}-q^{-}\right)}{\left[(\tilde{k}-\tilde{q})^{2}+\epsilon^{2}\right]} F(\tilde{q}, \ldots)=-\int d^{2} \tilde{q} \frac{\left(q^{-}\right)}{\left[(\tilde{q})^{2}+\epsilon^{2}\right]} F(\tilde{q}+\tilde{k}, \ldots)
$$

For a small neighborhood $\delta$ around the origin in the last integral, we have

$$
\int_{|\tilde{q}| \leqslant \delta} d^{2} \tilde{q} \frac{\left(q^{-}\right)}{\left[(\tilde{q})^{2}+\epsilon^{2}\right]}=0
$$

Thus there is no singular contribution.

Now, from equation (4.1) it follows that

$$
\begin{aligned}
& \int d^{2} \tilde{q} \tilde{\phi}_{s}\left(\tilde{q}, p_{3}\right)=-<\operatorname{meson}(p)|\bar{\psi}(0) \psi(0)| 0> \\
& \int d^{2} \tilde{q} \tilde{\phi}_{-}\left(\tilde{q}, p_{3}\right)=-<\operatorname{meson}(p)\left|\bar{\psi}(0) \gamma_{-} \psi(0)\right| 0>
\end{aligned}
$$

Assuming a scalar bound state exists, we get that the right hand side of the first of equations (5.6) is non-zero, while that of the second is zero. ${ }^{2}$ This in turn implies that $\tilde{\phi}_{s}\left(\tilde{k}, p_{3}\right)$ behaves like $\frac{1}{k_{s}^{2}}$, as follows from the first of equations (5.1). But then the integral on the left hand side of the first of equations (5.6) diverges. Hence a contradiction.

Similarly, we can rule out vector bound states as well.

\section{Bound-states in the bosonic theory}

Similar to the theory of a fundamental fermion coupled to a CS theory given in (2.1) one can formulate a theory of a scalar in the fundamental representation coupled to a CS theory of the following form

$$
S_{B}=\frac{i K_{B}}{4 \pi} \int d^{3} x \operatorname{Tr}\left[A d A+\frac{2}{3} A^{3}\right]+D_{\mu} \bar{\phi} D^{\mu} \phi+m_{B}^{2} \bar{\phi} \phi+\frac{1}{2 N_{B}} b_{4}(\bar{\phi} \phi)^{2}
$$

\footnotetext{
${ }^{2}$ Note that we are in the rest frame of the bound state, so no _ component for the matrix element of the current.
} 
where now one defines $\lambda_{B}=\frac{N_{B}}{K_{B}}$. It was further shown in [3] that in the Wilson-Fisher limit of

$$
b_{4} \rightarrow \infty, \quad m_{B} \rightarrow \infty, \quad \frac{4 \pi m_{B}^{2}}{b_{4}}=\left|c_{B}\right| \text { fixed }
$$

where $c_{B}^{2}$ is the mass $^{2}$ that appears in the full scalar propagator, the bosonic theory (6.1) is equivalent to the fermionic one (2.1). In [18] the $(2 \rightarrow 2)$ scattering amplitude of the bosonic theory was evaluated. In particular it was found that for the range

$$
-2 \pi \lambda_{B} c_{B}\left(4-\lambda_{B}\right) \geq b_{4} \geq-2 \pi c_{B}\left(4-\lambda_{B}^{2}\right)
$$

the scattering amplitude admits poles which correspond to particle anti-particle bound states in the singlet channel. Note that as the correspondence with the fermionic theory is only for $b_{4}$ tending to $\infty$, there will be no bound states in the fermionic case.

Furthermore, note that there are bound states in the bosonic theory also when there is no coupling to the gauge fields, namely in the limit of $\lambda_{B}=0$. The bound states are generated by the coupling $(\bar{\phi} \phi)^{2}$ alone in this case. Of course the coupling to gauge fields has an effect, as the relation among the parameters for the appearance of bound states now involves $\lambda_{B}$ as well.

\section{Confronting the results of $[19]$}

As mentioned in previous section, bound states in a Chern-Simons theory with scalars were considered [18]. They find bound states for certain values of the parameters. They also find duality relations with the fermionic theory. It turns out that the region of the scalar case, which is dual to the fermion case, has no bound states. Our results are in accordance with those.

On the other hand, a massless scalar bound state for the massive fermion case was claimed in [19], in contradiction with [18]. Note that in [19] the relation $m_{\text {bare }}=m\left(\frac{1}{\lambda}-\lambda\right)$ is employed, due to some assumed non-perturbative effect, and not (3.15). The latter, however, is the one derived in [1].

One may adopt the more general point of view of two parameters, $m_{\text {bare }}$ and full mass $m$, with not necessarily a relation between them, as in [19]. The origin of two mass parameters according to [19] comes from a contribution of a zero mode.

Adopting the two parameter approach, our relations (3.16) will have to change. The function $f_{0}$ is now

$$
p_{s} f_{0}=\lambda \sqrt{p_{s}^{2}+m^{2}}+m_{\text {bare }}
$$

Actually as in (3.14). The function $g_{0}$ will change too, but the relation

$$
g_{0}+f_{0}^{2}=\frac{m^{2}}{p_{s}^{2}}
$$

still holds, as in (3.10). 
Going over to the bound state equations, the relations (4.13) still hold, now with the two parameter $f_{0}$. But the proof of no bound states still goes through, as the asymptotic behavior of $f_{0}$ for large $p_{s}$ is unchanged.

So we get no bound states also in the two parameter case, unlike [19].

\section{Summary and open questions}

In this note we have shown that the theory of fermions in the fundamental representation of $\mathrm{U}(N)$ gauge symmetry coupled to a CS term at level $K$ in the large $\mathrm{N}$ large $\mathrm{K}$ limits such that $\lambda=\frac{N}{K}$ is fixed does not admit fermion anti-fermion bound states. This provides a stronger statement than the one in our previous publication [1] where we have shown that high mass bound states are forbidden. We derive this result by analyzing a 't Hooft like equation for the bound state wave-function. We showed that assuming a non-vanishing wave-function we get a contradiction in the limit of large "parton" momentum.

There are several open questions associated with results of this note:

- Analyzing 't Hooft like equations for the wave-functions of bound states for the theory with scalars in the fundamental representation instead of fermions. As was discussed in section 6 in the theory of scalars due to the scalar self coupling there are bound states. It is interesting to determine the spectrum of masses of the bound states using the technique employed in this note

- One can generalize the case discussed in this note to the case of several fermion multiplets with several different masses. The naive intuition is that also for those cases there are no ferimon anti-fermion bound states. This statement could be verified by explicit generalization of the statement of this note.

- One can investigate the question of what are the necessary condition for an interaction of fundamental fermions to admit a spectrum of bound states. It is well known that coupling of the fermions to a Yang Mills term would yield bound states. The question is what other type of interactions share the same property.

- A very natural question is what of all the results of the exact solution of the theory of fermions coupled to a CS term in 3d are relevant to theories of non-abelian fermions in four space-time dimensions.

\section{Acknowledgments}

We would like to thank Ofer Aharony for his comments on the manuscript and for insightful conversations, and Guy Gur-Ari for useful discussions. We would also like to thank the correspondence with W. Bardeen. J. S. acknowledges support of the "Einstein Center of Theoretical Physics" at the Weizmann Institute.

Open Access. This article is distributed under the terms of the Creative Commons Attribution License (CC-BY 4.0), which permits any use, distribution and reproduction in any medium, provided the original author(s) and source are credited. 


\section{References}

[1] Y. Frishman and J. Sonnenschein, Breaking conformal invariance - Large-N Chern-Simons theory coupled to massive fundamental fermions, JHEP 12 (2013) 091 [arXiv:1306.6465] [INSPIRE].

[2] S. Giombi, S. Minwalla, S. Prakash, S.P. Trivedi, S.R. Wadia et al., Chern-Simons Theory with Vector Fermion Matter, Eur. Phys. J. C 72 (2012) 2112 [arXiv:1110.4386] [INSPIRE].

[3] O. Aharony, S. Giombi, G. Gur-Ari, J. Maldacena and R. Yacoby, The Thermal Free Energy in Large-N Chern-Simons-Matter Theories, JHEP 03 (2013) 121 [arXiv:1211.4843] [INSPIRE].

[4] O. Aharony, G. Gur-Ari and R. Yacoby, Correlation Functions of Large-N Chern-Simons-Matter Theories and Bosonization in Three Dimensions, JHEP 12 (2012) 028 [arXiv:1207.4593] [INSPIRE].

[5] O. Aharony, G. Gur-Ari and R. Yacoby, D=3 Bosonic Vector Models Coupled to Chern-Simons Gauge Theories, JHEP 03 (2012) 037 [arXiv:1110.4382] [INSPIRE].

[6] G. Gur-Ari and R. Yacoby, Correlators of Large-N Fermionic Chern-Simons Vector Models, JHEP 02 (2013) 150 [arXiv:1211.1866] [INSPIRE].

[7] J. Maldacena and A. Zhiboedov, Constraining conformal field theories with a slightly broken higher spin symmetry, Class. Quant. Grav. 30 (2013) 104003 [arXiv: 1204.3882] [INSPIRE].

[8] J. Maldacena and A. Zhiboedov, Constraining Conformal Field Theories with A Higher Spin Symmetry, J. Phys. A 46 (2013) 214011 [arXiv:1112.1016] [INSPIRE].

[9] S. Giombi and X. Yin, On Higher Spin Gauge Theory and the Critical $\mathrm{O}(N)$ Model, Phys. Rev. D 85 (2012) 086005 [arXiv:1105.4011] [INSPIRE].

[10] S. Giombi and X. Yin, Higher Spin Gauge Theory and Holography: The Three-Point Functions, JHEP 09 (2010) 115 [arXiv:0912.3462] [INSPIRE].

[11] T. Takimi, Duality and higher temperature phases of large- $N$ Chern-Simons matter theories on $\mathrm{S}^{2} \times \mathrm{S}^{1}$, JHEP 07 (2013) 177 [arXiv: 1304.3725] [INSPIRE].

[12] S. Jain, S. Minwalla, T. Sharma, T. Takimi, S.R. Wadia et al., Phases of large-N vector Chern-Simons theories on $\mathrm{S}^{2} \times \mathrm{S}^{1}$, JHEP 09 (2013) 009 [arXiv: 1301.6169] [INSPIRE].

[13] S. Yokoyama, Chern-Simons-Fermion Vector Model with Chemical Potential, JHEP 01 (2013) 052 [arXiv: 1210.4109] [InSPIRE].

[14] S. Giombi and X. Yin, The Higher Spin/Vector Model Duality, J. Phys. A 46 (2013) 214003 [arXiv: 1208.4036] [INSPIRE].

[15] S. Banerjee, S. Hellerman, J. Maltz and S.H. Shenker, Light States in Chern-Simons Theory Coupled to Fundamental Matter, JHEP 03 (2013) 097 [arXiv:1207.4195] [INSPIRE].

[16] G. 't Hooft, A Two-Dimensional Model for Mesons, Nucl. Phys. B 75 (1974) 461 [InSPIRE].

[17] Y. Frishman and J. Sonnenschein, Non-perturbative Field Theory, from two dimensional conformal field theory to QCD in four dimensions, Cambridge monographs on mathematical physics, Cambridge University Press, Cambridge U.K. (2010).

[18] S. Jain, M. Mandlik, S. Minwalla, T. Takimi, S.R. Wadia et al., Unitarity, Crossing Symmetry and Duality of the S-matrix in large-N Chern-Simons theories with fundamental matter, arXiv:1404.6373 [INSPIRE].

[19] W.A. Bardeen, The Massive Fermion Phase for the $\mathrm{U}(N)$ Chern-Simons Gauge Theory in $D=3$ at Large $N$, JHEP 10 (2014) 039 [arXiv: 1404.7477] [INSPIRE]. 